\title{
Telescoping in dating naturally occurring events
}

\author{
CHARLES P. THOMPSON \\ Kansas State University, Manhattan, Kansas \\ JOHN J. SKOWRONSKI \\ Ohio State University at Newark, Newark, Ohio \\ and \\ D. JOHN LEE \\ Tabor College, Hillsboro, Kansas
}

\begin{abstract}
Telescoping effects in date estimation were examined in four diary studies. The data show that substantial telescoping can begin as soon as 8 weeks after an event occurs. These studies also found a slight, but typically nonreliable, tendency to make time expansion errors for recent events. Analyses of these data showed that telescoping cannot be attributed to the clarity-of-memory hypothesis proposed by Bradburn, Rips, and Shevell (1987) or to an artifact produced by guessing. An implicit strategy involving estimation of the number of intervening events was proposed to account for the results.
\end{abstract}

Memory errors are a major methodological problem in survey research. One type of memory error that has been given intensive scrutiny is the telescoping error: the tendency to assign a date to an event that is more recent than the actual date of occurrence. This error is rather troublesome because surveys are often aimed at establishing the frequency with which certain events occurred during a particular time period. For example, here are some timebounded questions from national surveys: "Now I'd like to ask some questions about crime. They refer only to the last 6 months..." (Bureau of Justice Statistics, 1985); "During the past year, about how many times did [you] see a medical doctor?" (Center for Health Statistics, 1985); and "During the past year, how many days has this condition kept you in bed all or most of the day?" (Center for Health Statistics, 1981).

There have been many studies on the telescoping problem (e.g., Gray, 1955; Neter \& Waksberg, 1964). Most of them seem to agree (see Skogan, 1981) that there is an overestimation of the frequency of events for a given time interval because people mistakenly import events that actually happened earlier. One interesting aspect of this literature is that telescoping is typically demonstrated by the apparent overestimation of the frequency of events occurring during a given time period (e.g., Loftus \& Marburger, 1983), rather than by errors in the estimated date of occurrence for specific events. The frequency measure seems somewhat indirect because the term telescoping clearly implies that subjects are erring in estimating the dates of occurrence of events.

A clear obstacle to assessing telescoping errors in terms of the date of occurrence of events lies in establishing the actual dates of occurrence of the events. "Public" events

Address correspondence to Charles P. Thompson, Department of Psychology, Bluemont Hall, Kansas State University, Manhattan, KS 66506. (such as the date of the bombing of the American Embassy in Beirut), for which the date is well known and/or obtainable from public records, can be used in an attempt to address this problem (Brown, Rips, \& Shevell, 1985). However, a long history of research on actor-observer differences in attribution suggests that actors and observers may encode and store information about an event in very different ways (see Monson \& Snyder, 1977). The use of public events has the disadvantage of being nonpersonal. Thus, it is quite possible that the use of public events will not satisfactorily address the question inherent in the original issue: What is the magnitude of telescoping in date estimation when people answer questions about their own lives?

The primary focus of this paper is to examine the magnitude of telescoping of personal events by using a procedure that establishes the exact date of occurrence for each personal event tested. The procedure is the diary method employed by Thompson (1982, 1985a, 1985b). In those experiments, subjects daily recorded brief descriptions of unique personal events for a period of about 3 months. During a test session that followed the diary-keeping period, those descriptions were read to subjects in a random order. The subjects attempted to date the events, and also rated how well they remembered the events. Because this procedure establishes the exact date of occurrence for each event, both the direction and magnitude of any dating error can be exactly established. Thus, this methodology allows researchers to assess, for any desired retention interval, the magnitude of telescoping or time expansion (i.e., evaluating an event as older than it actually is) errors in dating personal events.

The second focus of this paper is to evaluate a theoretical proposal regarding the processes underlying the phenomenon of telescoping. The diary procedure allows one to measure other event characteristics (e.g., affect, 
memorability). The possible effects of those characteristics on date estimation can then be determined. This feature of the diary procedure is particularly important in light of the recent hypothesized relationship between the clarity of memory and telescoping. Brown et al. (1985) noted that, because older memories are less detailed or clear than recent memories, the clarity of memory might serve as a cue for event age; that is, clearer memories would be judged as more recent and less clear memories would be judged as older. More recently, Bradburn, Rips, and Shevell (1987) suggested that telescoping might be produced by these well-remembered events' being judged as more recent. Because a subject's memory for an event can be easily assessed in the diary memory procedure, that procedure is well-suited to evaluate the clarity-ofmemory hypothesis.

For the benefit of the reader, we describe here our current strategy in diary memory studies. Because diary memory data is tedious to collect, we attempt to make the diary data as rich as possible so that it might be used in connection with other studies to make other points. The focus of the present study was on telescoping; however, we included ratings that allowed us to replicate an earlier study on pleasantness of the events. We also collected weekly data on the frequency of potentially common events (e.g., trips to the library, movies seen, longdistance telephone calls). We think it is helpful for our readers to know which sets of data involve a common group of subjects. At the same time, we think a single report on all aspects of the data would be unfocused and incoherent. Thus, we report only the data relevant to our main purpose. Furthermore, when possible, we use data from previous studies to buttress points of current interest.

\section{EXPERIMENT 1}

\section{Method}

Subjects. The subjects were 35 undergraduates at Kansas State University. All received class credit for participation.

Materials. The materials were recorded by the subjects and consisted of unique personal events that occurred during a 3-month period. They recorded one event each day, and the event records were collected once each week. The subjects were instructed that there were three restrictions on the events: (1) the events had to be unique (i.e., they were expected to occur no more than once during that semester), (2) they could not be embarrassing, and (3) they were to be described in three written lines or less.

Preratings. At the time an event was recorded, the individual recording the event predicted the memorability of the event on a 3-point scale, with 3 being extremely memorable and 1 being not very memorable. An extremely memorable event was defined as an event that would be remembered 1 year after it had occurred. A not very memorable event was defined as one that would probably be forgotten within 2 weeks.

The events selected for recording covered a wide range of topics that obviously differed in significance and memorability. Some events rated as not very memorable included the following: "Cleaned off the cobwebs on my bicycle and rode a couple of miles," "Ordered a teddy bear poster for Jodi," and "Planned this year's garden." By contrast, the following events were rated as extremely memorable: "Got accepted into the College of Veteri- nary Medicine" and "My roommate's father had a heart attack and died unexpectedly."

In addition to the predicted memorability ratings, the subjects rated the pleasantness of the event. They used a 7-point scale ranging from +3 for very pleasant to -3 for very unpleasant. Those data were collected to replicate data on pleasantness previously reported (Thompson, 1985a).

Procedure. Each of the 35 subjects generated events, as described, for a 12-week period. The events were collected on Wednesday of each week.

The memory test was given during the 13th week after recording began. Each participant was tested individually. The events were numbered sequentially, beginning with the first event recorded. The events were then read to the subject using one of two random sequences.

As each event was read, the subject first determined whether or not the event was unique. This procedure was used because the subjects obviously could not accurately date events that occurred more than once during the semester. The subjects had no problems making the uniqueness judgment. If the event was not unique, it was deleted, and the subject went on to the next event. If the event met the requirement of being unique, the subject rated how well the event was remembered on a 7-point scale. This scale was developed by Herrmann and Neisser (1978) and is as follows: not at all (1), barely at all (2), not so well (3), fairly well (4), very well (5), almost perfectly (6), and perfectly (7). The subject was advised that a 7 rating was very unusual: "This means that if the event involved a conversation, you could essentially recall the conversation wordfor-word." If the event was not remembered at all (rating 1), the subject went on to the next event. It would not make sense to ask for the date of occurrence for items that were not remembered. If the event was remembered, the subject attempted to estimate the date of occurrence of the event using a calendar containing no information other than date and day of the week.

The subjects began keeping their diaries about $21 / 2$ weeks into the semester. At the time of test, they were not given either the starting date for recording or the starting date of the semester. Judging from the frequent (and unsolicited) comments from the subjects, the vast majority of them did not know those dates, or the dates of other quite salient events (e.g., spring break). Because of the uncertainty about either starting date, errors in dating those oldest events could be telescoping or time expansion errors. Note that even if the subjects knew the starting date, the (minimum) 2-week block used in scoring events allows for errors in either direction. More important, both types of errors are found in the data.

Half the subjects began dating the events immediately. The other half began dating after half the events had been presented and given memory ratings. In the latter case, the subjects rated and dated events until all the events had been presented. At that point, the first half of the events were represented for dating. The re-presentation followed the same random order as the original presentation.

At the half-way point in dating events, all subjects were given the dates for two of their events located in the 4th and 9th weeks of the recording period. The event judged to be most salient during each of those 2 weeks was selected as a reference event. The dates of these personal events were given to the subjects on a $3 \times 5$ card, and the subjects were told to attempt to use those events as reference events in dating. Furthermore, the subjects were encouraged to add additional events (for which they knew the exact date) for use as reference events.

Neither the time at which subjects began dating nor the provision of reference events had any effect on the accuracy of dating events. Thus, those manipulations are not discussed further.

Comments on test memory ratings. Because memory ratings are not a standard procedure for measuring memory, we note the following three points about the memory ratings: (1) No natural memory study can duplicate a standard recall procedure. In a labora- 
tory study, the exact material to be recalled is known. Any attempt to abstract the most relevant material from an event will make assumptions about what "should" be remembered. (2) The memory rating is equivalent to cued recall, not to recognition. The short description elicits memory for all the characteristics of the event (e.g., the duration, the content, and the emotion) while describing, at best, a small subset of those characteristics. (3) The data strongly suggest that the rating procedure reflects recall. The memory rating data show the classical Ebbinghaus function and behave in other ways predicted by standard laboratory experiments on recall. These three points are discussed in some detail in earlier papers (Thompson, 1982, 1985a, 1985b).

\section{Results}

To measure the extent of telescoping, the 12-week recording period was divided into six 2-week intervals. The median signed dating error in days was calculated for each interval. Negative numbers indicate events that were thought to occur more recently than they did occur (telescoping), and positive numbers indicate events that were thought to occur earlier than they did occur (time expansion). Medians were used instead of means because all subjects occasionally made unusually large dating errors (as much as 71 days). These dating errors were entered into a one-way analysis of variance (ANOVA).

We used 2-week retention intervals to provide a reasonably fine-grained analysis of telescoping. However, those intervals produced far too many missing data points when crossed with a two-level memory rating split. Thus, to evaluate the clarity of memory hypothesis, the data for each subject were sorted into two 5-week blocks, excluding the most recent 2 weeks. A comment on the elimination of the most recent 2 weeks of data is in order. We found that subjects rarely made dating errors during the most recent week or two. Thus, any time expansion or telescoping effect would be reduced in magnitude (and possibly statistically eliminated) by the inclusion of those data.

The data were further sorted into well-remembered (memory ratings 5-7) and poorly remembered (memory ratings $2-4$ ) events. The median signed dating error was then calculated for each of the four cells for each subject. As noted above, the subjects occasionally made unusually large dating errors. Thus, to be conservative, any median based on less than three scores was replaced by the mean of the medians for that cell. Three medians were replaced in these data. Furthermore, 1 subject was eliminated from the analysis because he rated only one event as well-remembered. The resulting data were analyzed using a $2 \times 2$ repeated measures ANOVA.

To evaluate the reliability of any telescoping or time expansion, we used a trivial modification of the NewmanKeuls procedure. Specifically, we included a zero in the set of means and used the Newman-Keuls procedure to determine which means were reliably different from zero.

Telescoping. The analysis of the 2 -week interval data shows that a substantial amount of telescoping occurred for events in the two oldest intervals (see Table 1), producing a significant retention interval effect $[F(5,170)=$ $11.70, M S \mathrm{e}=30.22, p<.001]$. A subsequent Newman-
Table 1

Means of the Median Signed Dating Error (in Days)

in Experiments 1, 2, 3, and 4

\begin{tabular}{ccccccc}
\hline \multicolumn{6}{c}{ Mean Retention Interval (Weeks) } \\
\cline { 1 - 6 } & 1 & 3 & 5 & 7 & 9 & 11 \\
\hline
\end{tabular}

Median Signed Dating Error (days)

\begin{tabular}{lrrrrrr} 
Experiment 1 & .40 & 1.71 & .43 & .57 & -3.57 & -6.77 \\
Experiment 2 & .00 & .23 & -.07 & -1.40 & -2.73 & -3.97 \\
Experiment 3 & .52 & 1.17 & .39 & -.91 & -4.65 & -6.09 \\
Experiment 4 & & & & & & \\
$\quad$ Experienced Events & .50 & 1.28 & .44 & -3.47 & -5.94 & \\
Reported Events & 2.11 & .92 & .92 & -3.44 & -9.75 & \\
\hline
\end{tabular}

Keuls test shows that the two oldest intervals differed reliably from one another and from the other intervals. There were no reliable differences among the other intervals.

Clarity of memory and magnitude of telescoping. The magnitude of telescoping was unaffected by clarity of memory $(F<1)$. As before, there was reliable telescoping for older events $[F(1,33)=23.63, M S e=35.76$, $p<.001]$. Although the memory rating main effect was not reliable, there was a reliable retention interval $x$ memory rating interaction $[F(1,33)=4.15, M S e=$ 20.30, $p<.05$ ]. Those means are presented in Table 2 . However, a subsequent Newman-Keuls test showed that clarity of memory did not affect the amount of telescoping for older events, but both show reliable telescoping. Furthermore, there was no reliable time expansion for more recent events.

\section{Discussion}

The data suggest that substantial telescoping occurs for personal events by the time they are 2 months old. The

Table 2

Means of the Median Signed Dating Error (in Days) for Well-Remembered and Poorty Remembered Events in Experiments 1, 2, 3, and 4

\begin{tabular}{|c|c|c|}
\hline \multirow[b]{2}{*}{ Events } & \multicolumn{2}{|c|}{ Retention Interval } \\
\hline & Recent Events & Oid Events \\
\hline \multicolumn{3}{|c|}{ Experiment 1} \\
\hline Poorly remembered & 1.62 & -4.94 \\
\hline Well-remembered & .32 & -3.09 \\
\hline \multicolumn{3}{|c|}{ Experiment $2 *$} \\
\hline Poorly remembered & .00 & -2.03 \\
\hline Well-remembered & -.20 & -2.03 \\
\hline \multicolumn{3}{|c|}{ Experiment $3^{*}$} \\
\hline Poorly remembered & .48 & -3.39 \\
\hline Well-remembered & 1.70 & -3.96 \\
\hline \multicolumn{3}{|c|}{ Experiment 4} \\
\hline \multicolumn{3}{|l|}{ All Events } \\
\hline Poorly remembered & 2.24 & -4.49 \\
\hline Well-remembered & .61 & -2.86 \\
\hline \multicolumn{3}{|l|}{ Guesses removed } \\
\hline Poorly remembered & 2.50 & -3.80 \\
\hline Well-remembered & .53 & -1.52 \\
\hline
\end{tabular}

*The interaction was not statistically reliable. 
amount of telescoping increases with increasing retention interval. The data also suggest that modest time expansion occurs at very short retention intervals, although the effect is not reliable.

There is no support for the clarity-of-memory hypothesis. There was no reliable difference in telescoping for well-remembered and poorly remembered events. Indeed, the numerical difference for older events suggested more telescoping for poorly remembered than for wellremembered events.

It is always helpful to provide evidence that an effect is replicable. Accordingly, we used data from two previous experiments to evaluate the clarity-of-memory hypothesis and the amount of telescoping (or time expansion) that occurs during a 3-month period.

The method and results for the two experiments follow. Because the method in the two experiments essentially is identical to that used in Experiment 1, we note only the critical differences and refer the reader to the original papers for a complete description. The overall pattern of results will be assessed following presentation of both sets of results.

\section{EXPERIMENT 2}

\section{Method}

Subjects. The subjects were 30 undergraduates at Kansas State University. All received class credit for participation.

Materials, Preratings, and Procedure. The materials, preratings, and procedure were essentially identical to those used in Experiment 1. For a detailed description of the experiment and method, see Thompson (1985a).

\section{Results}

Telescoping. As in Experiment 1, each subject's median signed dating error within each of six 2-week intervals was entered into a one-way ANOVA. The results of this analysis, together with a subsequent Newman-Keuls test, were consistent with the results of Experiment 1. Date estimates for the oldest three intervals were telescoped, but were accurate (i.e., did not differ reliably from zero) for the most recent three intervals (see Table 1) $[F(5,145)=5.52, M S \mathrm{e}=16.01, p<.001]$.

Clarity of memory and magnitude of telescoping. As in Experiment 1, the data were sorted by retention interval (two 5-week blocks, excluding the most recent 2 weeks) and memory rating (ratings $2-4$ and 5-7). Seven medians were based on less than three estimates and were replaced with the mean for the appropriate cell.

Once again, clarity of memory did not affect the magnitude of telescoping $(F<1)$. As before, older events were telescoped more $(-2.03)$ than were more recent events $(-0.10)[F(1,29)=4.90, M S e=22.87, p<.05]$. The interaction was not reliable $(F<1)$. However, for comparison with other experiments, the means for the interaction are presented in Table 2. A Newman-Keuls test showed that there was no reliable telescoping for more recent events.

\section{EXPERIMENT 3}

\section{Method}

Subjects. The subjects were 23 undergraduates at Kansas State University. All received class credit for participation.

Materials, Preratings, and Procedure. The materials and relevant preratings were identical to those used in Experiment 1. With two exceptions, the procedure was also identical to that used in Experiment 1. The two exceptions were that the events were collected on Tuesday of each week and the memory test was given during the 14th week following the beginning of recording. For a more detailed description of the experiment and method, see Thompson (1985b, Experiment 2).

\section{Results}

Telescoping. As in the first two experiments, each subject's median signed dating error within each of six 2 week intervals was entered into a one-way ANOVA. The results of this analysis, together with the subsequent Newman-Keuls test, were consistent with the results of the first two experiments. Date estimates were telescoped forward for events in the oldest two intervals, but were accurate for events in the four most recent intervals (see Table 1) $[F(5,110)=10.26, M S e=20.66, p<.001]$.

Clarity of memory and magnitude of telescoping. As in the other two experiments, the data were sorted by retention interval (two 5-week blocks, excluding the most recent 2 weeks) and memory rating (ratings 2-4 and 5-7). Two medians were based on less than three estimates and were replaced with the mean for the appropriate cell.

Once again, clarity of memory did not affect the magnitude of telescoping $(F<1)$. As before, older events were telescoped more $(-3.67)$ than were more recent events $(1.09)[F(1,22)=11.92, M S e=43.72, p<.01]$. The Newman-Keuls test showed that the telescoping for the older events was reliable, but the time expansion for the more recent events was not reliable. The interaction was not reliable $(F=1.14)$. Again, for comparison with other experiments, the interaction means are shown in Table 2.

\section{Discussion}

The results of Experiments 2 and 3 completely replicated the results of Experiment 1. These data also showed substantial telescoping for personal events by the time the events were 2 months old. The amount of telescoping increased with an increase in retention interval, and there was no reliable time expansion at short retention intervals. Once again, there was no support for the clarity-ofmemory hypothesis, and there was no reliable difference in telescoping for well-remernbered and poorly remembered events.

\section{EXPERIMENT 4}

The three previous experiments demonstrated substantial telescoping but provided no support for the hypothesis that well-remembered events will be estimated as hav- 
ing occurred more recently than poorly remembered events.

Although the clarity-of-memory hypothesis was not supported by the data, the possibility remains that such effects might exist but be obscured by other effects. Specifically, there is some suggestion of time expansion as well as telescoping in the dating estimates, and it is possible that both errors in estimation result from guessing. That is, when subjects are uncertain about the date of an event, they may try to minimize the error in their estimate by placing the date of the event near the middle of the recording period. Subjects are very accurate in dating recent events so that not many recent events would be so placed. However, guessing those few events would produce a tendency for apparent time expansion for recent events. The tendency to guess would increase with increasing retention interval. Thus, events beyond the midpoint of the recording interval would show apparent telescoping, and that apparent telescoping would increase with increasing retention interval.

The telescoping and time expansion errors produced by guessing might obscure any clarity-of-memory effects. To eliminate the possibility that clarity-of-memory effects are obscured by guessing, one must eliminate the events for which the date is guessed. We do that in two ways in the following experiment: First, we had subjects indicate the strategy they used to date the event. If they selected "guess" as the strategy, then we eliminated those events from the analysis. Second, we had subjects rate the confidence with which they dated the events, and we analyzed only those events for which they were very confident of the date.

The main purpose for which this experiment (Lee, 1987) was conducted was to evaluate the distinction between experienced and reported events in personal memory (Larsen, 1987). However, since date estimation was one of the memory measures taken to compare the two types of events, telescoping and time expansion were considered as well.

\section{Method}

Subjects. The 36 subjects were members of two psychology classes at Tabor College, a small liberal arts college in Kansas.

Materials. The materials were unique personal events recorded by the subjects over a 10- to 11-week period. Each day the subjects recorded two unique events, an experienced event and a reported event. Experienced events were defined as those that were "directly experienced or first-hand personal experiences." Reported events were defined as those "events that someone else has told you about." As in the previous experiments, the subjects were instructed that the events were to be nonembarrassing and briefly described.

Preratings. At the time the events were recorded, the recorder predicted the memorability of the event on the 3-point scale described in Experiment 1.

Procedure. Individual memory tests were administered during the 11 th and 12th weeks after recording began. The subjects recorded events up to the day before they were tested. A sample of 80 events (40 experienced and 40 reported) was taken from the 10 weeks preceding the test day. Eight events, four of each type, were selected from each week. Due to the nature of class schedules, the events recorded on Mondays, Wednesdays, Thursdays, and Saturdays were taken as representative events. If an item was not recorded on one of these days, then the item from the following day was used, with the exception that Tuesday events were used to replace missing events from Wednesday.

The 80 events were presented in one of two random orders. The procedure for testing each event was the same as in the previous experiments except for the following changes: (1) After the subjects estimated the date of an event, they were asked to rate how confident they were about the date they had selected. A 5-point confidence scale was used, with 1 identified as $a$ wild guess, 2 as not very sure, 3 as maybe right, 4 as pretty sure, and 5 as very confident. (2) The subjects were asked to indicate when they had selected a date by guessing ("picked a date essentially at random").

\section{Results}

Four different analyses were performed on these data to evaluate hypotheses about telescoping. The first evaluated the extent of telescoping for both experienced and reported events. The second analysis evaluated the clarityof-memory hypothesis. The third analysis was one of two evaluating the hypothesis that telescoping is produced by placing events for which the date is a guess near the center of the interval during which the events occurred. In this analysis, all events for which the subjects selected "guess" as their strategy for dating were eliminated. The fourth analysis was an even more stringent evaluation of the artifact hypothesis. In this analysis, all events except those with the two highest confidence ratings were eliminated.

Telescoping. The median signed dating error was calculated for each subject for each of five 2 -week retention intervals separately for experienced and reported events.

There is a clear effect of telescoping shown in these data. There was a reliable effect of retention interval $[F(4,140)=39.82, M S e=28.66, p<.001]$. There was also a reliable retention interval $\times$ event type interaction $[F(4,140)=4.05, M S \mathrm{e}=18.42, p<.01]$. The means for each retention interval are shown separately for experienced and reported events in Table 1. A NewmanKeuls post-test showed that both experienced and reported events show reliable telescoping for the two longest retention intervals. There was no reliable time expansion for either type of event.

Clarity of memory and magnitude of telescoping. As in the previous experiments, the data for each subject were sorted by two 5 -week retention intervals and by poorly remembered (ratings 2-4) versus well-remembered (ratings 5-7) events. This sorting was done separately for experienced and reported events. The median signed dating error was calculated for each cell. Fifteen cells (5\%) had no entries and were filled with the mean for the appropriate group.

Once more, telescoping was demonstrated in these data. There was a reliable effect of retention interval $[F(1,35)$ $=38.15, M S e=49.05, p<.001]$. The interaction of retention interval with memory rating was also reliable $[F(1,35)=6.36, M S e=30.12, p<.02]$. The means for the interaction also are presented in Table 2. Post hoc tests on the means showed that there was reliable tele- 
scoping for both well-remembered and poorly remembered events (i.e., they both differed from zero). However, the two types of events did not differ from one another. Time expansion was not reliable for either poorly remembered events or well-remembered events. No other main effect or interaction was statistically significant $(F \mathrm{~s}<1)$.

Telescoping with guesses removed. The dating estimates that subjects said were guesses were removed, and the median signed dating error was recalculated for each subject. As in the above analysis, the data were sorted by two 5 -week retention intervals and by poorly remembered versus well-remembered events.

As noted earlier, telescoping (or time expansion) may be a result produced by subjects placing events for which the date is uncertain near the middle of the interval during which events were collected. Furthermore, because there was more guessing with poorly remembered events, the numerically greater telescoping for those events might also be attributable to guessing. It follows that the clarityof-memory hypothesis might be supported when guessed events are removed.

These data suggest that telescoping cannot be solely attributed to guessing. The analysis showed a reliable effect of retention interval $[F(1,35)=27.67, M S e=45.30$, $p<.001]$ and a reliable interaction of retention interval with memory rating $[F(1,35)=13.61, M S e=23.96$, $p<.001]$. The means for the interaction are also presented in Table 2. Subsequent Newman-Keuls tests showed that well-remembered events showed neither reliable telescoping nor reliable time expansion. By contrast, poorly remembered events showed both effects. No other main effect or interaction was reliable $(F \mathrm{~s}<1.3)$.

In short, the clarity-of-memory hypothesis fares even worse when guessed events are removed. In direct contradiction to the hypothesis, poorly remembered events show reliable telescoping and well-remembered events do not.

Telescoping with confident dating estimates. Pure guessing has been eliminated as the only reason for telescoping. However, it is possible that telescoping occurs when subjects are not confident in their estimates of the date of occurrence. To check that possibility, median signed dating errors were calculated using only events with the two highest confidence ratings. As before, medians were calculated for each subject for two 5-week retention intervals separately for experienced and reported events. The well-remembered versus poorly remembered distinction was eliminated because too few subjects dated poorly remembered events with great confidence. Even with that factor eliminated, 23 cells $(16 \%)$ had no entries and were filled with the mean for the appropriate group.

Reliable telescoping occurs even for events that are dated quite confidently. There was a reliable main effect of retention interval $[F(1,35)=9.87, M S e=12.50, p<$ .01]. Subsequent Newman-Keuls tests showed that there was reliable telescoping for old events $(-1.64)$ but no reli- able time expansion for recent events (.21). No other main effect or interaction was statistically reliable $(F s<1)$.

Telescoping with confident dating estimates removed. Although the important point is that telescoping occurs even when only events dated quite confidently are considered, the complementary analysis is of some interest. We would expect substantial telescoping and, quite possibly, reliable time expansion to occur when subjects are not confident in their estimates of the date of occurrence. To check that possibility, median signed dating errors were calculated using only events with the three lowest confidence ratings. As before, medians were calculated for each subject for two 5-week retention intervals separately for experienced and reported events. The well-remembered versus poorly remembered distinction was not included for two reasons. First, we wanted the analysis to directly complement the analysis reported above. Second, a preliminary analysis showed no effect of clarity of memory $(F<1)$.

As expected, both telescoping and time expansion occur for events that are dated with little confidence. There was a reliable main effect of retention interval $[F(1,35)$ $=57.45, \mathrm{MSe}=35.90, p<.001]$. Subsequent Newman-Keuls tests showed that there was reliable telescoping for old events $(-5.38)$ and reliable time expansion for recent events (2.19). The event type $\times$ retention interval interaction was also significant $[F(1,35)=4.24$, $M S e=14.15, p<.05]$. That interaction is shown in Table 3. As can be seen, the magnitude of the difference between old and recent events is greater for reported than for experienced events. The main effect of event type was not reliable $(F<1)$.

\section{Discussion}

These data support the view that telescoping cannot be attributed solely to subjects' placing events in the middle of the time period when they are forced to guess the date of those events. Telescoping was demonstrated when events for which subjects reported guessing the date were removed from the analysis. However, telescoping was demonstrated only for poorly remembered events in the analysis with guesses removed. It is not at all surprising to see telescoping eliminated for those events for which the student both does not guess the date of the event and remembers the event very well.

In short, the data of Experiment 4 replicate the telescoping effect found in the three previous experiments.

Table 3

Means of the Median Signed Dating Error (in Days) for Events Dated with Low Confidence Ratings (Ratings 1, 2, or 3)

Retention Interval

\begin{tabular}{lcc} 
& \multicolumn{2}{c}{ Retention Interval } \\
\cline { 2 - 3 } & Recent Events & Old Events \\
\hline Experienced Events & -1.75 & 4.53 \\
Reported Events & -2.64 & 6.22 \\
\hline
\end{tabular}

Note-The means are shown separately for experienced and reported events as well as recent and old events. 
There is substantial telescoping of personal events by the time the events are 2 months old. As in the previous experiments, these data show no support for the clarity-ofmemory hypothesis. In fact, the analysis of the data for which subjects were quite confident of the date directly contradicts that hypothesis.

Consistent with the other three experiments, the overall data and data with guesses removed show no reliable time expansion. Although there was a numerical trend suggesting time expansion in most of the data, that trend was never reliable. Unlike telescoping, which occurs for all types of events, the pattern of results for time expansion shows that it occurs only for poorly remembered events. In short, these data show both telescoping and time expansion. Telescoping occurs for all types of events, whereas time expansion occurs only for poorly remembered events.

\section{GENERAL DISCUSSION}

The dating of events usually involves estimation. With the exception of important recurring events (such as birthdays and holidays) and extraordinary personal or historical events (e.g., the date the atom bomb was dropped on Hiroshima), the dates of events are rarely stored in memory.

Survey research (e.g., Skogan, 1981) suggests that subjects often underestimate the age of an event, a bias termed telescoping. One characteristic of survey research is that it uses a retrospective approach in which subjects are asked to recall a limited number of behaviors whose dates can be verified (e.g., reported crimes). In contrast, we were interested in determining the extent and direction of dating errors for a wide range of personal events. The diary-keeping procedure allows the collection and analysis of this kind of information.

Four diary-keeping experiments showed the same pattern of telescoping errors over a 3-month period. When the 3-month period is divided into 2-week blocks, subjects showed substantial telescoping in the two longest intervals. In the three studies using only experienced events, there was no reliable time expansion effect for short retention intervals. However, in the experiment including reported events, subjects also showed reliable time expansion, but only for the poorly remembered recent events.

\section{The Clarity-of-Memory Hypothesis Fails}

We have shown that subjects use a variety of strategies to date events when the exact date cannot be directly retrieved (Thompson, Skowronski, \& Lee, 1987). Which of these multiple strategies for estimating the dates of events could account for the telescoping effect? The data from all four experiments provide an appropriate test for the proposal that telescoping is produced by date estimates based on the clarity of memory (Bradburn et al., 1987; Brown et al., 1985). All four experiments failed to support that hypothesis. In fact, the numerical difference be- tween well-remembered and poorly remembered events was consistently in the opposite direction to that predicted by the clarity-of-memory hypothesis.

Subjects report that they rarely use the clarity-ofmemory strategy to date events (Thompson et al., 1987). Taken alone, those reports would leave open the possibility that clarity of memory is used implicitly, rather than explicitly, as a strategy to date events. However, taken together with the data reported here, it seems unlikely that clarity of memory is a significant factor in producing telescoping in dating events.

\section{Two Alternative Hypotheses}

We suggest two potential processes that might affect the degree of telescoping. One process is hypothesized to produce telescoping, whereas the other simply affects the magnitude of telescoping. After describing the processes, we will point out that they lead to opposite predictions about the relative magnitude of telescoping.

First, we speculate that telescoping might be produced by estimation of the date based on the number of events remembered (implicitly or explicitly) to have occurred during the retention interval. Several studies indicate that the number of events (e.g., Block, 1974) or the number of significant changes (e.g., Block, 1978) affects the perceived duration of the interval. As the number of intervening events increases, the perceived duration of the interval increases.

The intervening-event strategy has been proposed in the time estimation literature that deals, for the most part, in retention intervals of minutes and seconds (see Fraisse, 1984). However, the strategy seems completely applicable to the much longer retention intervals observed in the present experiments. Briefly stated, the suggestion is that subjects attempt to estimate the age of an event that cannot otherwise be dated by estimating the number of events that have intervened since the target event occurred. Event memory declines with time, and we assume that subjects attempt to adjust for that memory loss. However, we also assume that subjects underestimate their memory loss at longer retention intervals and, as a result, underestimate the length of the interval. Therefore, for older events, there will be significant telescoping, and that telescoping will increase with an increase in retention interval. By contrast, recent events are well-remembered and accurately dated. Thus, subjects may assume that any events that cannot be dated precisely must have occurred at a moderate retention interval. If this is true, one would predict a modest time expansion effect for events at short retention intervals.

Second, we suggest that telescoping will be greatly reduced as individuals develop a temporal frame of reference (Robinson, 1986). Robinson eloquently pointed out that people in different occupations (e.g., students, farmers, professional athletes) have different temporal frames of reference. However, there are common characteristics of those temporal reference schemata. Most important, the year is segmented into temporal periods (e.g., 
spring semester, summer break) that are characterized by contrasting activities (e.g., classes vs. summer job).

We suggest that telescoping will be reduced to the degree that a person has an elaborate temporal frame of reference. As an individual's temporal frame of reference becomes more elaborate, that individual typically has more temporal reference points to which many events can be related. Hence, dating becomes more accurate and telescoping is reduced. Note that a temporal frame of reference affects (i.e., reduces) telescoping but does not speak to the process(es) underlying telescoping.

\section{Contrasting Predictions from the Hypotheses}

Individuals obviously must learn their relevant temporal frame of reference. Thus, newcomers to a particular occupation (e.g. , college student, professional athlete) typically will have a less elaborate, and probably less accurate, temporal frame of reference. Both characteristics will lead to less accurate dating (and, presumably, more telescoping) for those just starting with a particular temporal frame of reference.

The opposite result is predicted by the intervening-event hypothesis. Newcomers to an occupation will have more memorable events and more significant changes during an initial period in their temporal frame of reference. Thus, the hypothesis would predict less telescoping for newcomers than for those well-established in the occupation.

A fairly strong test of the two proposed processes could be achieved by contrasting freshmen and junior college students during the fall semester. Freshmen are just beginning and are adapting to a temporal frame of reference that differs significantly from that of their high schools (e.g., classes on Monday, Wednesday, and Friday). Juniors have presumably become quite knowledgeable about the college time frame. We think that a diary study contrasting juniors and freshmen might be a useful next step in our attempt to understand the processes underlying telescoping.

\section{REFERENCES}

BLock, R. A. (1974). Memory and the experience of duration in retrospect. Memory \& Cognition, 2, 153-160.

BLock, R. A. (1978). Remembered duration: Effects of event and sequence complexity. Memory \& Cognition, 6, 320-326.

Bradburn, N. M., Rups, L. J., \& Shevell, S. K. (1987). Answering autobiographical questions: The impact of memory and inference on surveys. Science, 236, 158-161.
Brown, N. R., Rips, L. J., \& Shevell, S. K. (1985). The subjective dates of natural events in very-long-term memory. Cognitive Psychology, 17, 139-177.

Bureau of Justice Statistics (1985). Criminal victimization in the United States, 1983 (NCJ Publication No. 96459). Washington, DC: U.S. Government Printing Office.

Fraisse, P. (1984). Perception and estimation of time. In M. R. Rosenzweig \& L. W. Porter (Eds.), Annual Review of Psychology, 35, 1-36.

Gray, P. G. (1955). The memory factor in social surveys. Journal of the American Statistical Association, 50, 344-363.

Herrmann, D. J., Neisser, U. (1978). An inventory of everyday experiences. In M. M. Gruneberg, P. E. Morris, \& N. Sykes (Eds.), Practical aspects of memory (pp. 35-51). New York: Academic Press.

LARSEN, S. (1987, August). Memory for reported events. Paper presented at the Second Intemational Conference on Practical Aspects of Memory, Swansea, Wales.

LEE, D. J. (1987). Memory for naturally-occurring events: Experienced versus reported events and telescoping in dating estimation. Unpublished doctoral dissertation, Kansas State University, Manhattan.

Loftus, E. F., Marburger, W. (1983). Since the eruption of Mt. St. Helens, has anyone beaten you up? Improving the accuracy of retrospective reports with landmark events. Memory \& Cognition, 11 , 114-120.

Monson, T. C., \& SNyder, M. (1977). Actors, observers, and the attribution process: Toward a reconceptualization. Joumal of Experimental Social Psychology, 13, 89-111.

National Center for Health Statistics (1981). Plan and opera. tion of the second national health and nutrition examination survey 1976-80 (DHHS Publication No. 81-1317). Washington, DC: U.S. Govemment Printing Office.

National Center for Health Statistics (1985). The national health interview survey design, 1973-84, and procedures, 1975-83 (DHHS Publication No. 85-1320). Washington, DC: U.S. Government Printing Office.

Neter, J., * WAksBerg, J. (1964). A study of response errors in expenditures data from household interviews. Journal of the American Statistical Association, 59, 17-55.

RoBINSON, J. A. (1986). Temporal reference systems and autobiographical memory. In D. C. Rubin (Ed.), Autobiographical memory (pp. 159-188). New York: Cambridge University Press.

SKOGAN, W. G. (1981). Issues in the measurement of victimization (Report No. NCJ-74682). Washington, DC: Bureau of Justice Statistics.

Thompson, C. P. (1982). Memory for unique personal events: The roommate study. Memory \& Cognition, 10, 324-332.

Thompson, C. P. (1985a). Memory for unique personal events: Effect of pleasantmess. Motivation \& Emotion, 9, 277-289.

Thompson, C. P. (1985b). Memory for unique personal events: Some effects of the personal schema. Human Learning: Joumal of Practical Research \& Applications, 4, 267-280.

Thompson, C. P., Skowronski, J, J., \& LeE, D. J. (1987, August). Reconstructing the date of a personal event. Paper presented at the Second International Conference on Practical Aspects of Memory, Swansea, Wales.

(Manuscript received July 13, 1987; revision accepted for publication January 4, 1988.) 